\title{
Atypical Levels of Heavy Metals, Creatinine, Haptoglobin, and Ferritin Found in Amyotrophic Lateral Sclerosis Outpatients vs. Healthy Controls: Possible Utility as a Diagnostic Biomarker
}

\author{
Taruna Ikrar ${ }^{1,3 * \#, ~ D a v i d ~ A ~ S t e e n b l o c k ~}{ }^{2 \#}$, Terawan Agus Putranto ${ }^{3}$ \\ ${ }^{1}$ International School of Biomedical Sciences, Pacific Health Sciences University, USA \\ ${ }^{2}$ Steenblock Research Institute, USA \\ ${ }^{3}$ Cellcure Center, The Indonesia Army and Presidential Central Hospital, Indonesia \\ ${ }^{\#}$ These authors (T.I. \& D.A.S.) contributed equally to this work.
}

*Corresponding author: Prof. Taruna Ikrar, MD., PhD. at Cellcure Center, The Indonesia Army and Presidential Central Hospital, (RSPAD Gatot Subroto), Jln. Abdul Rahman Saleh No. 24, Jakarta 10410, Indonesia; International School of Biomedical Sciences, Pacific Health Sciences University, BakerField, California 93309, USA, Phone: +62-21-3441008, +62-213840702 Ext 5005, Fax: +62-21-350619, E-mail: taruna.ikrar@pacifichealthu.org

\begin{abstract}
Heavy metal toxicity has been conjectured as a possible risk factor for and player in amyotrophic lateral sclerosis (ALS) and other neurodegenerative disorders. To test for a correlational relationship or linkage, we conducted a retrospective study of 54 sALS and 26 healthy volunteers who agreed to serve as controls, all of whom were seen at the Personalized Regenerative Medicine Clinic (San Clemente, California USA) between 2011 and 2016.

We evaluated clinical laboratory findings including heavy metal content in blood and urine samples, along with serum creatinine, ferritin and haptoglobin levels, by means of inductively coupled plasma optical spectrometry and instrumental activation analysis. Disease progression, assessed using the Revised ALS Functional Rating Scale (ALS-FRS-R), was generally associated with mercury, lead, cadmium, arsenic and nickel burden. In our analysis, we found evidence of an association between ALS morbidity risk and elevated levels of mercury, lead, cadmium, arsenic, and nickel.

Our findings implicate and support a linkage between relatively high levels of heavy metals, elevated serum ferritin and haptoglobin levels, and reduced urine creatinine level and sporadic amyotrophic lateral sclerosis. By virtue of this the authors argue that these may serve as a biomarker to help distinguish clinically ALS from other, symptom similar neurogenerative conditions.
\end{abstract}

\section{Keywords}

ALS, Neurodegeneration, Heavy metals, Mercury, Lead, Cadmium, Nickel, Arsenic, Creatinine

\section{Introduction}

Amyotrophic lateral sclerosis (ALS) is typically a lethal neurologic disease characterized by progressive muscular weakness with atrophy and fasciculation. Although the etiology of ALS is unclear, previous epidemiologic studies have suggested an association with exposure to various environmental chemicals and metals environment [1-3].

In addition, many researchers have found that autoimmune pathways and mechanisms are implicated in ALS, which is compelling as environmental factors including xenobiotics such as chemicals, drugs and heavy metals, have been implicated in the development of various autoimmune diseases. Interestingly, autoantibodies occur in systemic and organ specific autoimmune diseases and sometimes are detected prior to their onset and by virtue of this can be used as predictive markers with respect to these. Some are disease specific markers and are employed to establish a diagnosis, to record progression and predict the outcome Utility as a Diagnostic Biomarker. Int J Pathol Clin Res 5:091. doi.org/10.23937/2469-5807/1510091 Accepted: May 29, 2019: Published: May 31, 2019

Copyright: (c) 2019 Ikrar T, et al. This is an open-access article distributed under the terms of the Creative Commons Attribution License, which permits unrestricted use, distribution, and reproduction in any medium, provided the original author and source are credited. 
of various diseases $[4,5]$. In addition, the extant scientific literature clearly demonstrates that heavy metals induce autoimmunity in a variety of animal models, and reports exist that link metal exposure to the development of autoimmunity in man include epidemiological studies, occupational exposure to them, and a high incidence of side-effects following treatment with metal chelators and colloidal gold $[3,6]$.

Metals in nature occur bound to sulfur groups in metal ores in the ground. When extracted for industrial use, they are purified and thereby lose their chemical stability. Some transition metals such as iron, cobalt, zinc, selenium, molybdenum, magnesium, chromium, manganese and copper are essential for life. Others, such as mercury, lead, nickel, cadmium, palladium, and arsenic are widely used in industry and in various implants but are toxic at certain levels in humans [7-9].

While the pathophysiology of sporadic ALS remains unclear, some environmental factors including heavy metals has been considered as an etiologic player in the disease [6,10-12].

The aim of this retrospective study was to determine whether significant heavy metal levels exist in a population of sALS outpatients vs. healthy controls, and, where present, to touch on mechanisms as to how they might play a role in this insidious disease. The authors also looked at creatinine, haptoglobin, and ferritin levels in both sALS patients and healthy controls (All of which are believed influenced by the ALS disease process or as a consequence of "downstream" effects of the disease).

\section{Materials \& Methods}

\section{Description of patients}

ALS subjects were recruited through the Personalized Regenerative Medicine Clinic (San Clemente, California USA) with the following inclusion criteria: (1) Age greater than 18 years; (2) Probable (lab test-supported), or definite ALS which meets the revised El Escorial criteria; (3) Able to provide informed consent; (4) Had complete medical charts on-file at the aforementioned clinic. The study was approved by the Institutional Review Board at the nonprofit Steenblock Research Institute (SRI). All participants provided written informed consent.

Fifty-four (54) sporadic ALS patients (28 men and 26 women) charts were selected for inclusion in the retrospective in the study. Twenty-six (26) gender matched healthy controls were also included.

Patient mean (SD) age is 62.3 (10.7) years (range, 4378 years).

\section{Determination of urinary metals, creatinine, and serum of blood samples}

All laboratory samples collected from the fifty-four (54) ALS patients were analyzed by LabCorp Laboratory, Inc. The normal reference ranges for metals were ob- tained from published data and standards [13,14]. After an initial chart review, stored serum samples from the 54 participants with ALS and twenty-six (26) healthy control volunteers, all of whom were included in a separate ALS observational study that ran from 2011 to 2016, were analyzed for urine creatinine, metals levels, serum ferritin, and haptoglobin. These samples were sent to the Pathology Laboratory at LabCorp Laboratory, Inc and analyzed using an electro chemiluminescent immunoassay or enzyme-linked immunosorbent assay (ELISA), ELECSYS 2010 (Roche Diagnostics, Indianapolis, IN) or Creatinine ICP-MS by Jaffe Method. The serum haptoglobin levels were measured in a hospital laboratory using a Beckman Coulter Unicel DXC by combining an antihaptoglobin antibody with the haptoglobin molecule, forming an insoluble complex [15]. This antigen-antibody complex was measured by optical absorbance at 340 nanometers using the turbidimetry principle. The serum haptoglobin was reported in milligrams per deciliter $(\mathrm{mg} / \mathrm{dL})$.

\section{Statistical Analysis}

The data collected was expressed as mean \pm standard error of the mean (SEM), with differences between groups determined by ANOVA with Student's t-test using GraphPad InStat. P $<0.05$ was considered statistically significant.

\section{Results}

\section{Heavy metals intoxication}

It is well documented in the literature that the physiologic damage wrought by mercury toxicity is magnified by the presence of other heavy metals such as cadmium, lead, nickel and arsenic.

In light of the foregoing, we analyzed clinical data on fifty-four (54) sALS cases which revealed significantly elevated mercury concentrations $(16.91 \pm 4.23 \mu \mathrm{g} / \mathrm{g})$ compared with the control group $(1.88 \pm 0.5 \mu \mathrm{g} / \mathrm{g}$, reference range $<2 \mathrm{ug} / \mathrm{g}$ ), All positive findings were statistically significant compared to the controls $(\mathrm{P}<$ 0.0001 ) Figure 1.

In one published study researchers reported that neuronal damage occurs in adults and children after poisoning with mercury compounds [16], although mercury poisoning in ALS patients with a history of occupational or other exposure has been rarely reported [17]. It is nonetheless true that environmental pollution involving mercury has led to an increased concern over the possibility that accumulated neurologic exposure is causing neuronal damage in some people. In this study, we found elevated mercury levels in all fifty-four (54) sALS patients. In addition, their ALS-related symptoms decreased following chelation therapy. What is intriguing being that the vast majority of these sALS patients showed evidence on computed tomography (CT) scans of degenerative pathology and a history of spinal inju- 


\section{MERCURY}

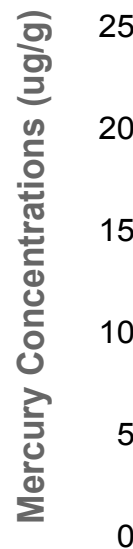

25

20

15

10

5

0

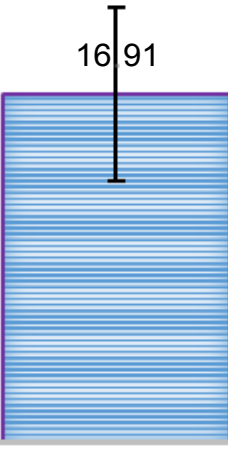

ALS
1.88

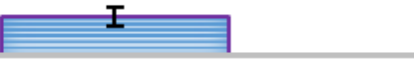

Control

$P<0.0001 ; N=54$

Figure 1: In addition, it has been suggested that environmental toxins could be a risk factor for amyotrophic lateral sclerosis (ALS) with mercury being implicated in a dose-dependent fashion.

\section{LEAD}

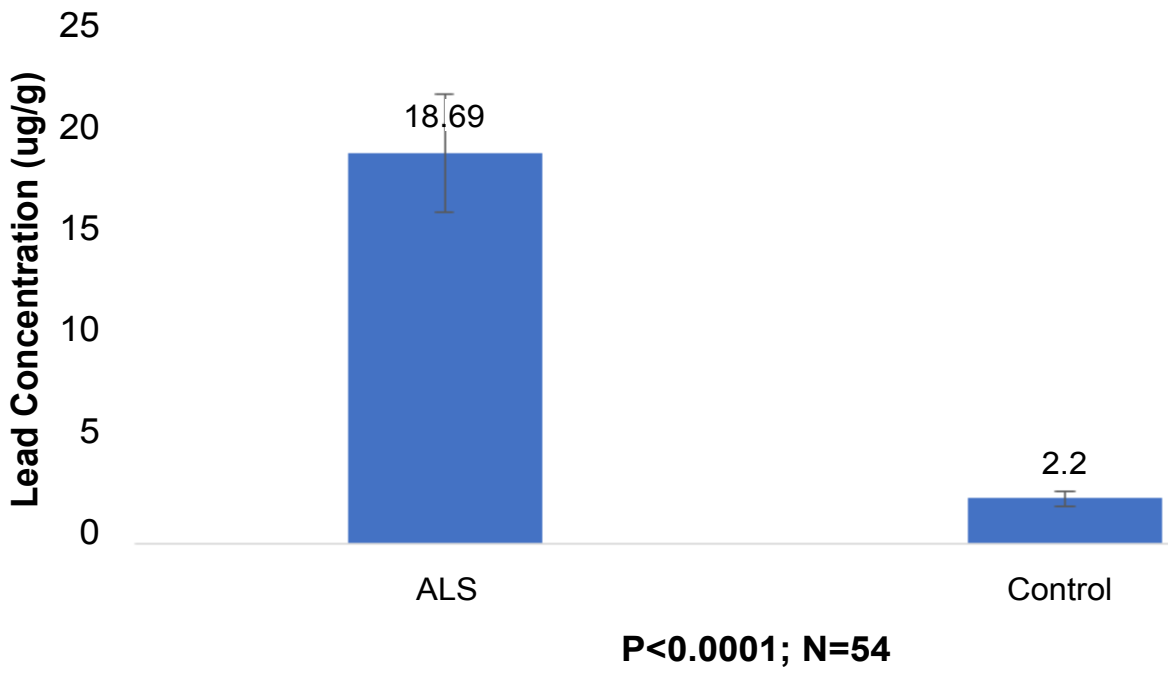

Figure 2: The relation of lead exposure in ALS has been well documented by researchers. For men and women combined, exposure to lead was associated with their development of ALS.

ry and (in many instances) reinjury to the original injury site (Something that could create breeches in the blood-spinal cord barrier that admit neurotoxic misfolded proteins, pro-inflammatory cytokines, activated monocytes, heavy metals bound to specific proteins and so on, into the central nervous system [18]). There were also many reports of peripheral neuropathy, tremor, and psychiatric symptoms including confusion and depression among this population which is symptomatically and clinically consistent with mercury toxicity [19].

In this study, lead concentrations were high (18.69 $\pm 2.1 \mu \mathrm{g} / \mathrm{g})$ in the ALS group compared with $(2.2 \pm 0.7$ $\mu \mathrm{g} / \mathrm{g}$ ) the control group (reference range $<2 \mu \mathrm{g} / \mathrm{g}$ ), P $<0.0001$, Figure 2 . This suggests a strong association between elevated levels of lead and sALS in our sample.

In one study, it was reported that high levels of cadmium, a sign of exposure to heavy metals, was found in the brain tissue of deceased ALS patients. The effects of cadmium on enzyme systems that mediate neurotoxicity in motor neuron disease suggest a causeeffect relationship between cadmium and ALS [20]. In the current study, our data revealed significantly elevated cadmium concentrations in the sALS group $(2.39 \pm 0.4 \mu \mathrm{g} / \mathrm{g})$ compared to the control group $(0.44 \pm$ $0.03 \mu \mathrm{g} / \mathrm{g}, \mathrm{P}<0.01$, Figure 3).

The resulting inflammation is modest compared to that of cadmium, although nickel induced strong TNF $\alpha$ production $[19,21]$. Nickel and arsenic were also 


\section{CADMIUM}

3

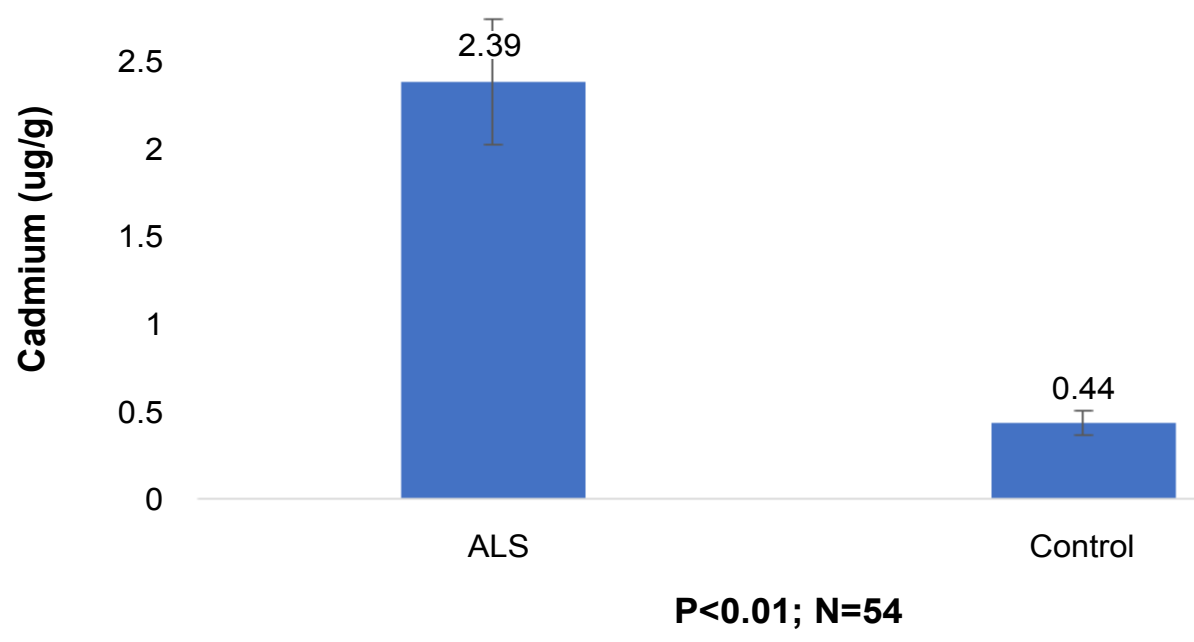

Figure 3: Cadmium impairs the blood-brain barrier, reduces levels of brain copper-zinc (Cu-Zn) superoxide dismutase (SOD), and enhances the excitotoxicity of glutamate via up-regulation of glutamate dehydrogenase and down-regulation of glutamate uptake in glial cells.

\section{NICKEL}

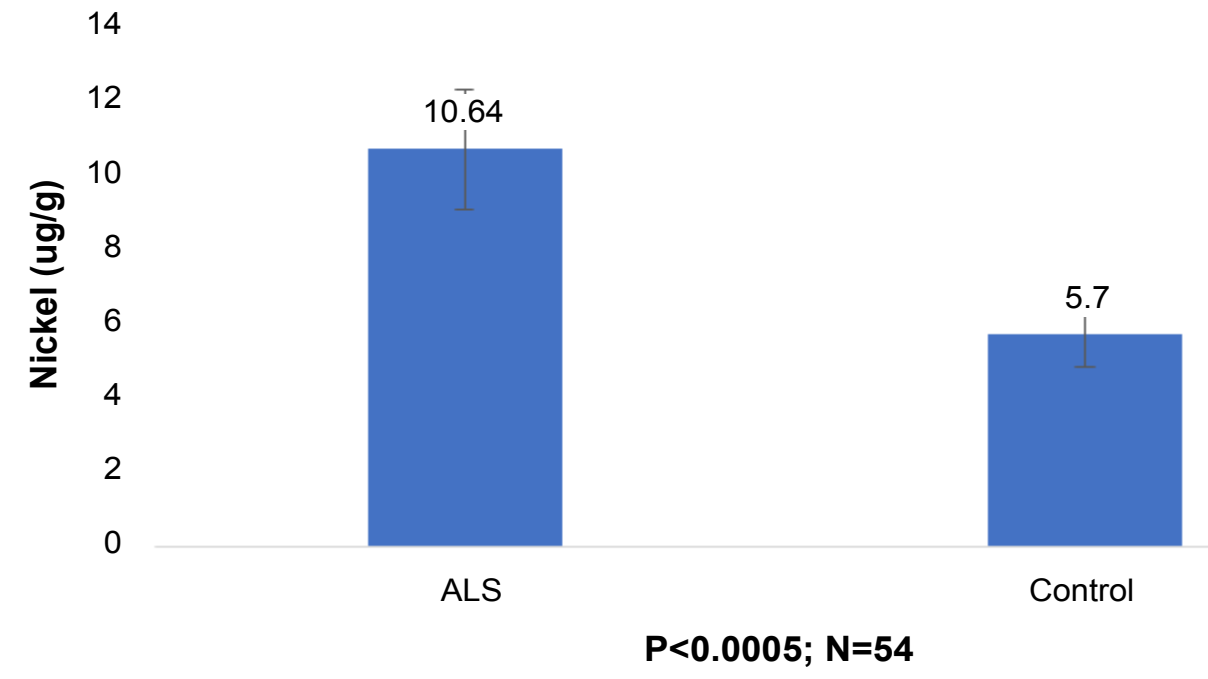

Figure 4: Nickel induced cell activation has been linked to increases in ferritin and haptoglobin levels.

found to induce strong phosphorylation of IкBa. Since inflammation produced by nickel might be relevant in SALS, we investigated the activation of human THP-1 cells. Laboratory tests revealed nickel concentrations in the sALS group $(10.64 \pm 2.1 \mu \mathrm{g} / \mathrm{g})$ to be significantly higher than in the healthy control group $(5.7 \pm 1.1 \mu \mathrm{g} / \mathrm{g}$, $P<0.0001$, Figure 4).

Arsenic inhibited translocation of NFs into axonal neurites in culture and increased perikaryal NF phosphoepitopes $[6,7]$. Folate deprivation potentiated the impact of arsenic on these activities, while supplementation with S-adenosyl methionine (SAM) attenuated the impact of folate deprivation on arsenic neurotoxicity [22]. Our data revealed an arsenic concentration in the sALS group $(35.58 \pm 5.3 \mu \mathrm{g} / \mathrm{g}$ ) that was very high compared to the control group $(3.08 \pm 0.46 \mu \mathrm{g} / \mathrm{g}$, $\mathrm{P}<$ 0.005 , Figure 5).

\section{Increased ferritin and haptoglobin levels in the blood of ALS patients}

The results of hematological analyses are reported. The levels of all hematological parameters except for lymphocytes and album non-significantly differed between sexes. All values were normally distributed within each sex.

The levels of ferritin in blood serum from these participants are shown (Figure 6) with gender producing no significant differences in ferritin $(P=0.3)$ and haptoglobin $(p=0.90)$ levels: The blood ferritin (male, $[325.5 \pm 9.3 \mathrm{ng} / \mathrm{mL}$; female, [330.2 $\pm 0.1 \mathrm{ng} / \mathrm{mL}], \mathrm{p}=$ 


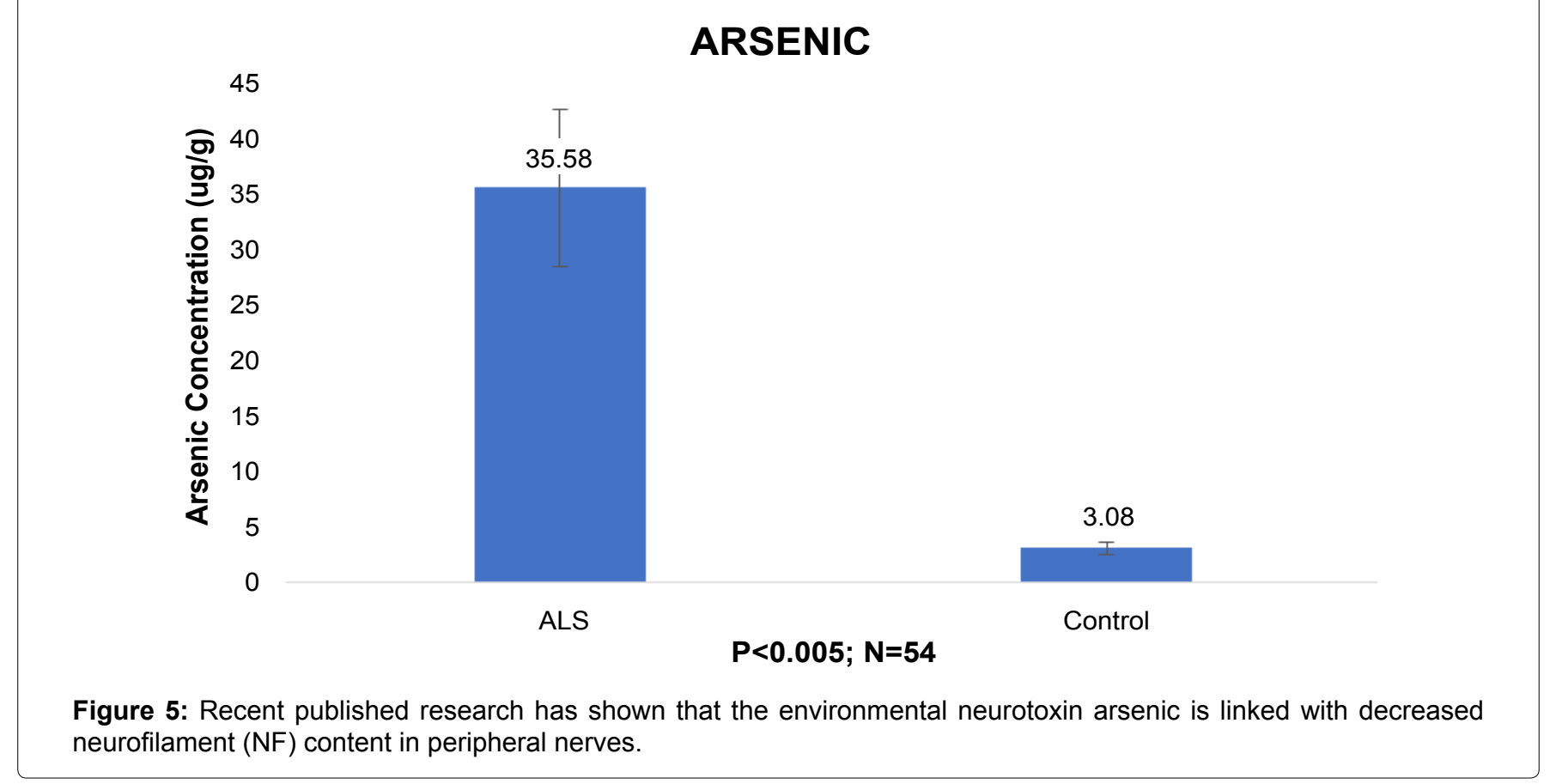

neurofilament (NF) content in peripheral nerves.

\section{Serum Ferritin}

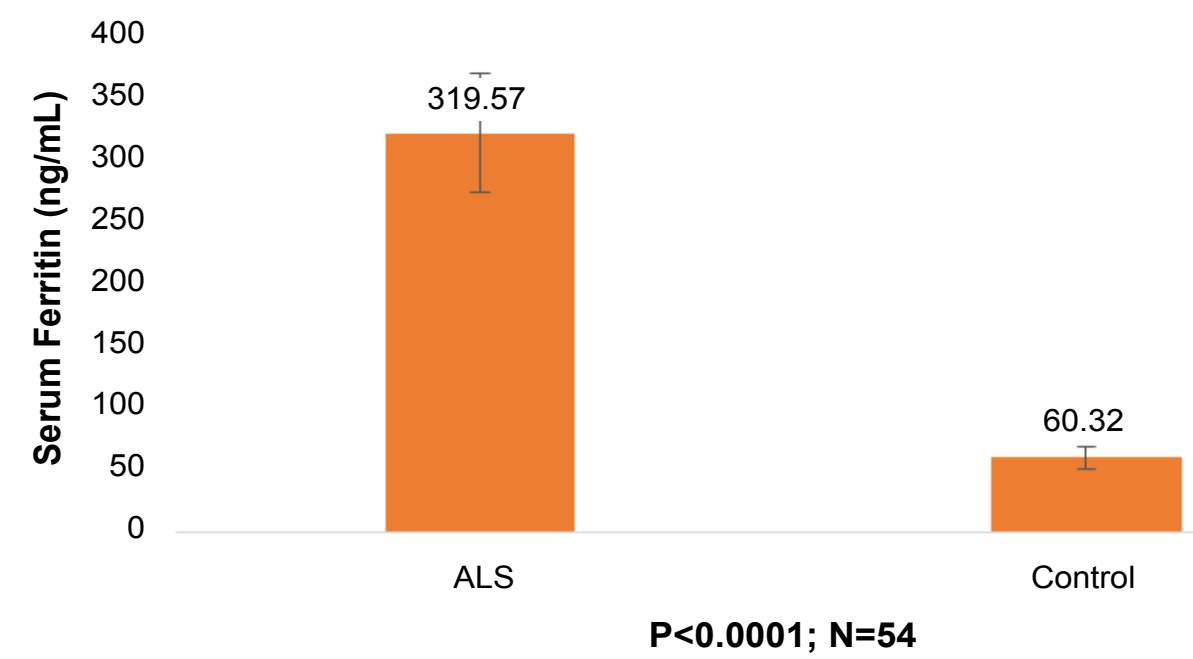

Figure 6: The increased levels of blood ferritin and haptoglobin were further examined in the 54 sALS patients compared with 26 controls using ELISA.

0.16), Haptoglobin (male, $[198.2 \pm 7.1 \mathrm{ng} / \mathrm{mL}]$; female, $[199.1 \pm 8.1 \mathrm{ng} / \mathrm{mL}], \mathrm{p}=0.16)$.

The ferritin level in the blood of ALS patients (319.57 $\pm 47.9 \mathrm{ng} / \mathrm{mL}$ ) was increased compared with the controls $(60.32 \pm 12.1 \mathrm{ng} / \mathrm{mL})(\mathrm{p}<0.0001)$, with the ferritin level in the ALS group showing a significant elevation compared to the control group, Figure 6.

The haptoglobin quotient, a marker of blood-brain barrier (BBB) function, was significantly higher in the ALS patients $(204.8 \pm 30.7 \mathrm{mg} / \mathrm{dL}$ ) than the controls (34 $\pm 5.1 \mathrm{mg} / \mathrm{dL})(P<0.0001)$, Figure 7 .

Haptoglobin is a protein synthesized in the liver that binds with the globin $\alpha$-chains of hemoglobin $A, F, S$, or C. Haptoglobin does not bind methemoglobin, heme, or unusual forms of hemoglobin in which the $\alpha$-chain is missing. The haptoglobin-hemoglobin complex is rapidly removed from circulation by the reticuloendothelial system to prevent/minimize hemoglobin loss and to conserve iron [14,21,23,24].

Ferritin levels were significantly increased in ALS patients compared to controls $(p<0.001)$, while no differences in the levels of serum iron, transferrin, iron saturation or total iron binding capacity were found. The increased ferritin level in the sporadic ALS patients may reflect a general increase in stored iron or be a consequence of ongoing muscle and motor neuron degeneration [25].

In one published study, haptoglobin is depicted as an acute phase protein and its inhibitory activity on pros- 


\section{Serum Haptoglobin}
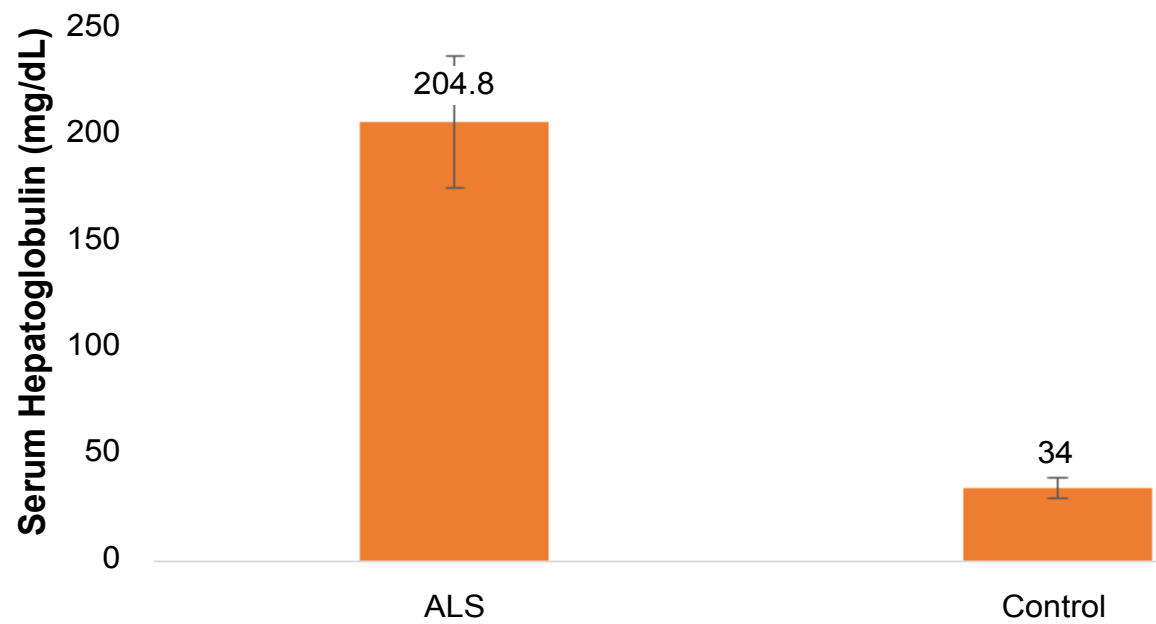

$\mathrm{P}<0.0001 ; \mathrm{N}=54$

Figure 7: We analyzed the results using univariate analyses for other hematological factors, however only ferritin concentrations and haptoglobin levels were significantly elevated with respect to ALS compare to controls.

\section{Creatinine Level}

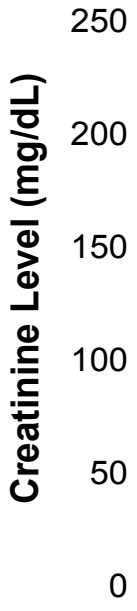

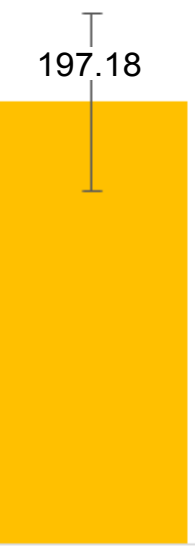

Control

\section{$\mathrm{P}<0.005 ; \mathrm{N}=54$}

Figure 8: Our data showed a correlation between urine creatinine and ALS in the study patient population.

taglandin synthetase and cathepsin B were proposed as part of the regulation of an inflammatory reaction. Its inhibitory effects on lymphocyte blastogenesis and monocyte chemotaxis at concentrations corresponding to those in clinical inflammatory states suggests its involvement in the immunological response. A sensitization of motor neuron disease lymphocytes to neuronal membrane components released by denervated muscle and the cytotoxic activities of ALS plasma against erythrocytes has been demonstrated in the laboratory. These factors reacted with antibodies present in ALS plasma known to suppress terminal axonal sprouting and subsequent reinnervation of skeletal muscle $[15,26]$. If, in fact, haptoglobin is involved in the regulation of any part of the immunological response, one may interpret the observed association between haptoglobin and motor neuron disease with some confidence. That is, the functional properties of haptoglobin may, in combination with other factors, be involved in the pathogenesis of ALS [24].

\section{Decreased urine creatinine}

A comparison of urine creatinine from ALS and controls are shown in Figure 8. The urinary creatinine levels in the ALS group was significantly elevated compared to controls $(P<0.005)$. The figure below shows the results of the creatinine levels of ALS patients compared to controls which signal that the latter had a significantly lower level of urinary creatinine. 
A total of fifty-four (54) sporadic ALS patients and twenty-six (26) gender-matched healthy controls were included. The Revised ALS Functional Rating Scale (ALSFRS-R) was used to assess the motor function status of the SALS patients. Urine creatinine levels were significantly lower in the sALS patients $(66.46 \pm 9.9 \mathrm{mg} /$ $\mathrm{dL}$ ) compared with the controls $(197.18 \pm 29.6 \mathrm{mg} / \mathrm{dL}$ ), $(P<0.005)$, Figure 8. Low urine creatinine levels are of concern as this may indicate deceased kidney functioning or severe muscle loss due to disease. Creatinine is a waste by-product that is created when muscles break down and metabolize creatinine, and is filtered from the blood by the kidneys and excreted through the urine. The levels seen in the SALS patients may indicate dysfunction of the glomerularus [27].

\section{Correlation as illustrated by a sALS Clinical Case}

A 58-year-old man presented to the Personalized Regenerative Medicine Clinic with a history of weakness in his left arm and shoulder, with discomfort and difficulty dressing himself during the past month and a half. Initially, he had attributed his issues to a prior rotator cuff injury. He then noted a progressive shrinking in the muscles of his left arm and hand with decreased grip strength and the development of uncomfortable 'charley horses' in his left leg.

The results of a neurological examination showed atrophy in the left biceps and deltoid and the left first dorsal interossei muscle. fasciculations were noted in the left forearm and left first dorsal interossei. An electrodiagnostic study performed the same week showed low amplitudes in the left upper extremity motor nerve-muscle action potentials with intact sensory nerve action potential responses. There were fibrillation potentials and positive sharp waves in the left deltoid, triceps, biceps, flexor carpi radialis, tibialis anterior, and bilateral gastrocnemius medial heads. His tongue showed a discrete firing pattern without abnormal resting activity. Sensory testing, coordination, and gait were within normal limits.

The results of neuroimaging studies of the spine revealed age-related degenerative joint and disc disease with spondylosis. A cervical spine MRI demonstrated some mild discogenic changes with moderate chronic cord deformity, but no active cord compression at C4-5, cord signal abnormality, and significant stenosis.

Further laboratory investigations revealed an immunoglobulin E total $(766 \mathrm{IU} / \mathrm{mL})$, serum ferritin $(724 \mathrm{ng} /$ $\mathrm{mL})$, haptoglobin $(500 \mathrm{mg} / \mathrm{dL})$, fibrinogen activity (344 $\mathrm{mg} / \mathrm{d})$. urine creatinine $(27.5 \mathrm{mg} / \mathrm{dL})$, and extremely high levels of various toxic heavy metals (Mercury 45 $\mu \mathrm{g} / \mathrm{g}$, lead $26 \mu \mathrm{g} / \mathrm{g}$, cadmium $7 \mu \mathrm{g} / \mathrm{g}$, arsenic $110 \mu \mathrm{g} / \mathrm{g}$, and nickel $23 \mu \mathrm{g} / \mathrm{g}$ ).

The patient's symptoms progressed to encompass weakness in the left shoulder with increasing weakness in the left arm. Examination showed atrophy in the bilat- eral spinet (left > right) along with persistent atrophy in the left deltoid and biceps. Fasciculations were noted in the upper extremity muscles in a scattered distribution, predominately proximally. Our diagnosis corresponded with the El Escorial criteria classification parameters [6].

\section{Discussion}

In this retrospective study, we analyzed data from fifty-four (54) sALS patients and twenty-six (26) healthy controls who were outpatients at the Personalized Regenerative Medicine Clinic (San Clemente, California USA). We found a positive association between heavy metals concentration, creatinine level, haptoglobin and ferritin levels and SALS morbidity. In addition, clinical symptomatology including compromised motor functioning in SALS patients was found to correlate in a dose dependent fashion with their heavy metals levels.

If the concentration of heavy metals and SALS is causal, it follows that otherwise healthy people with significantly elevated levels of heavy metals would be at an increased risk of developing ALS. Of course, it is possible that the positive association of heavy metals in this study's ALS patients was due to chance or residual confounding and thus represented no causal relationship [28].

In support of a putative link between heavy metal load and ALS:

Heavy metal exposure has been linked to the development of autoimmunity in humans in various epidemiological studies, plus reports of occupational exposure to metals, as wells as a high prevalence of side-effects following treatment with metal chelators and colloidal gold. Metals in ionic form reach cell membranes attached to circulating blood proteins, particularly the water-soluble component of lipoproteins $[29,30]$. It is this feature that allows ionic metals to exchange freely between lipoproteins and cell membrane ligands, including red blood cells. The hemoglobin of red blood cells is particularly rich in $\mathrm{SH}$ groups which further explains how ionic metals reach the various cell membranes via the blood. Since metals in ionic form are lipophilic, they readily pass through the blood brain barrier. For example, mercury readily oxidizes in the brain and nervous tissue to its ionic form, where the ionic mercury then binds with the $\mathrm{SH}$ groups of cell membranes, protein and brain enzymes [22].

The toxic effects of metals are mediated through free radical formation, cell membrane disturbance or enzyme inhibition, among other biochemical mechanisms. By binding to cell membranes, metals alter the membrane charge, which may result in changed membrane permeability, calcification and cell death. Metals also bind to mitochondria, thereby impairing cellular respiration. Depending on genetically determined detoxification systems, an individual may tolerate exposure to toxic metals before showing adverse effects [31]. 
The immunological effects of metals are either nonspecific such as immunomodulation or antigen specific such as allergy and autoimmunity. Metals may act as immunosuppressants (cytostatically) or as immunoadjuvants (non-specific activation of the immune system). One example of immunomodulation is the ability of metals to modify cytokine production in vitro and in vivo. Another example is the enhancement of the intensity and duration of antigen-specific IgE responses by mercury [23]. Metals may also induce allergic reactions in genetically susceptible individuals. It is anticipated that cellular reactions triggered by metals may operate elsewhere in the body where they are deposited. It has been shown that metals like zinc and cadmium regulate translation of ferritin by modulating binding of Iron regulatory protein 1 (IRP1) to the Iron-regulatory element (IRE) in the 5'UTR of ferritin. It is possible that lead acts on a similar pathway to impact ferritin synthesis [30].

Experimental evidence has demonstrated that chronic exposure to heavy metals such a lead and mercury tends to have a deleterious effect on motor neurons. Several case-control studies have examined the risk of ALS with exposure to lead, mercury, or heavy metals, with significant increased degeneration of motor neurons in those so exposed [17]. Mercury $(\mathrm{Hg})$, lead $(\mathrm{Pb})$, cadmium (Cd), and arsenic (As), nickel (Ni) are also recognized neurotoxins in children that particularly affect neurodevelopment and intellectual performance [25].

The data from this retrospective study revealed that all fifty-four (54) SALS patients had high levels of heavy metals.

Also, in this study the authors found that serum ferritin levels were elevated in all our SALS patients. Clinically, this is often associated with hemochromatosis and inflammatory stress. In the later case, stress induced inflammation is induced by cytokines such as interleukin-1 (IL-1) and other biochemical players. In a transgenic mutation superoxide dismutase (SOD1) mouse model of motor neuron disease, neural ferritin expression was increased in response to increased oxidative stress [15] Defects in ferritin metabolism are possibly involved in the etiology of other neurodegenerative deficits, including ALS.

The finding of decreased creatinine and increased ferritin in the study's SALS patients may represent a chronic disease phase or iron metabolism dysregulation. The role of creatinine and ferritin in ALS needs to be studied further, as does the role of creatinine and ferritin and other proteins involved in iron metabolism especially with respect to heavy metals intoxication that affect cellular oxidative stress levels [31].

Urine creatinine and haptoglobin have been found to be independent biomarkers of disease severity in men and women with amyotrophic lateral sclerosis (ALS) in various studies, with our study revealing that lower levels of urine creatinine and elevated levels of haptoglobin denoting a more serious disease stage as well as being predictive of a significant rate of progression. They also demonstrated sensitivity and specificity values at predicting morbidity that paralleled "the best established prognostic factors" for ALS, such as forced vital capacity, age, and scores on the ALS Functional Rating Scale-Revised.

As such, it is postulated from this study's findings that creatinine and haptoglobin are reliable and easily detectable blood and urine markers that correspond to the severity of motor dysfunction in ALS and could be used in helping with prognosis at the time of diagnosis.

In addition, study data revealed that serum creatinine levels were significantly lower in ALS patients than in controls $(p<0.005)$, something that argues for a role for creatinine in ALS pathophysiology and points to a correlation between serum creatinine and ALS. This study also found that sALS patients with low serum creatinine levels in had the most severe motor.

And, interestingly with respect to haptoglobin, one published study found that the myelin nerve damage observed in multiple sclerosis (MS) may be partly mediated through the long-term release and degradation of extracellular haemoglobin $(\mathrm{Hb})$ and the products of its oxidative degradation. The protein and acute phase reactant haptoglobin ( $\mathrm{Hpt}$ ) binds extracellular $\mathrm{Hb}$ as a first line of defence, and can serve as a vascular antioxidant $[16,19]$.

In conclusion, our results suggest that heavy metals, creatinine, haptoglobin, and ferritin are associated with the clinical severity and pathogenesis of ALS. And extrapolating from study findings, heavy metals burden plus increased ferritin and haptoglobin and reduced urine creatinine should serve as a reliable "collective" biomarker signature that will help clinicians distinguish ALS from other forms of neurologic pathology.

\section{Declaration of Interest}

The authors report no conflicts of interest. The authors alone are responsible for the content and writing of the paper.

\section{Conflict of Interest Statement}

The authors declare that the research was conducted in the absence of any commercial or financial relationships that could be construed as a potential conflict of interest. Authors claim no conflicts of interest related to the research described in the manuscript.

\section{References}

1. Song S, Miranda, Braun L, Meyer K, Frakes, et al. (2016) Major histocompatibility complex class I molecules protect motor neurons from astrocyte-induced toxicity in amyotrophic lateral sclerosis. Nat Med 22: 397-403.

2. Haidet-Phillips AM, Hester ME, Miranda CJ

(2011) 
Astrocytes from familial and sporadic ALS patients are toxic to motor neurons. Nat Biotechnol 29: 824-828.

3. Fogh I, Lin K, Tiloca C, Rooney J, Gellera C, et al. (2016) Association of a locus in the CAMTA1 gene with survival in patients with sporadic amyotrophic lateral sclerosis. JAMA Neurol 73: 812-820.

4. Gupta S, Ahern K, Nakhl F, Forte F (2011) Usefulness of haptoglobin levels to evaluate hemolysis in recently transfused patients. Adv Hematol 2011: 1-4.

5. Gupta S, Ahern K, Nakhl F, Forte F (2011) Clinical usefulness of haptoglobin levels to evaluate hemolysis in recently transfused patient. Adv Hematol 2011: 1-4.

6. Quandt SA, Jones BT, Talton JW, Whalley LE, Galván L, et al. (2010) Heavy metals exposures among Mexican farmworkers in eastern North Carolina. Environ Res 110: 83-88.

7. Xiang DH, Jiang $H$, Fu R, Zhai H, Shi $Y$, et al. (2008) Molecular dissection of ALS-associated toxicity of SOD1 in transgenic mice using an exon-fusion approach. Hum Mol Genet 17: 2310-2319.

8. Kiernan MC, Vucic S, Cheah B, Turner MR, Eisen A, et al (2011) Amyotrophic lateral sclerosis. Lancet 377: 945-955.

9. Frohlander N, Forsgren L (1988) Haptoglobin groups in motor neuron disease. J Neurol Neurosurg Psychiatry 51: 440-442.

10. Huang YC, Wu YR, Tseng MY, Chen YC, Hsieh SY, et al. (2011) Increased prothrombin, apolipoprotein A-IV, and haptoglobin in the cerebrospinal fluid of patients with huntington's disease. PLoS One 6: e15809.

11. Steenblock DA, Ikrar T, Antonio AAS, Wardaningsih E, Azizi MJ (2018) Amyotrophic Lateral Sclerosis (ALS) Linked to Intestinal Microbiota Dysbiosis \& Systemic Microbial Infection in Human Patients: A Cross-Sectional Clinical Study. Int J Neurodegener Dis 1: 1-4

12. Pamphlett $R$, Kum Jew $S$ (2016) Age-related uptake of heavy metals in human spinal interneurons. PLoS One 11: e0162260.

13. Chi Fru E, Rodríguez NP, Partin CA, Lalonde SV, Andersson $P$, et al. (2016) Cu isotopes in marine black shales record the great oxidation event. Proc Natl Acad Sci U S A 113: 4941-4946.

14. Bozzoni V, Pansarasa O, Diamanti L, Nosari G, Cereda C, et al. (2016) Amyotrophic lateral sclerosis and environmental factors. Funct Neurol 31: 7-19.

15. Garzillo EM, Miraglia N, Pedata P, Feola D, Lamberti M (2016) Risk agents related to work and amyotrophic lateral sclerosis: An occupational medicine focus. Int J Occup Med Environ Health 29: 355-367.

16. Cavaleri F (2015) Review of Amyotrophic Lateral Sclerosis, Parkinson's and Alzheimer's diseases helps further define pathology of the novel paradigm for Alzheimer's with heavy metals as primary disease cause. Med Hypotheses 85: 779-790.

17. Oskarsson B, Horton DK, Mitsumoto H (2015) Potential environmental factors in amyotrophic lateral sclerosis. Neurol Clin 33: 877-888.

18. Ikrar T (2018) Amyotrophic Lateral Sclerosis: New Suggestions of Pathophysiology and Treatments. Progress and Communication in Sciences 3: 9-18.

19. Chattopadhyay M, Nwadibia E, Strong CD, Gralla EB, Valentine JS, et al. (2015) The Disulfide bond, but not zinc or dimerization, controls initiation and seeded growth in amyotrophic lateral sclerosis-linked $\mathrm{Cu}, \mathrm{Zn}$ superoxide dismutase (SOD1) fibrillation. J Biol Chem 290: 3062430636.

20. Shi Y, Acerson MJ, Shuford KL, Shaw BF (2015) Voltageinduced misfolding of zinc-replete ALS mutant superoxide dismutase-1. ACS Chem Neurosci 6: 1696-1707.

21. Wang MD, Little J, Gomes J, Cashman NR, Krewski D (2017) Identification of risk factors associated with onset and progression of amyotrophic lateral sclerosis using systematic review and meta-analysis. Neurotoxicology 16: 101-130.

22. Garzillo EM, Miraglia N, Pedata P, Feola D, Sannolo N, et al. (2015) Amyotrophic lateral sclerosis and exposure to metals and other occupational/environmental hazardous materials: State of the art. G Ital Med Lav Ergon 37: 8-19.

23. Farrés M, Piña B, Tauler R (2016) LC-MS based metabolomics and chemometrics study of the toxic effects of copper on Saccharomyces cerevisiae. Metallomics 8: 790-798.

24. Furukawa Y, Anzai I, Akiyama S, Imai M, Cruz FJ, et al. (2016) Conformational disorder of the most immature $\mathrm{Cu}$, $\mathrm{Zn}$-superoxide dismutase leading to amyotrophic lateral sclerosis. J Biol Chem 291: 4144-4155.

25. Bocca B, Forte G, Oggiano R, Clemente S, Asara Y, et al. (2015) Level of neurotoxic metals in amyotrophic lateral sclerosis: A population-based case-control study. J Neurol Sci 359: 11-17.

26. Doyle CM, Rumfeldt JA, Broom HR, Sekhar A, Kay LE, et al. (2016) Concurrent increases and decreases in local stability and conformational heterogeneity in $\mathrm{Cu}, \mathrm{Zn}$ superoxide dismutase variants revealed by temperaturedependence of amide chemical shifts. Biochemistry 55: 1346-1361.

27. Imounan F, Lahbouje S, Hsaini Y, Regragui W, Ait Ben Haddou el $\mathrm{H}$, et al. (2015) Clinical and environmental aspects of amyotrophic lateral sclerosis in moroccan population: a study of 60 cases. Tunis Med 93: 365-370.

28. Lee JK, Shin JH, Gwag BJ, Choi EJ (2015) Iron accumulation promotes TACE-mediated TNF- $\alpha$ secretion and neurodegeneration in a mouse model of ALS. Neurobiol Dis 80: 63-69.

29. Friedman A, Arosio P, Finazzi D, Koziorowski D, GalazkaFriedman $J$ (2011) Ferritin as an important player in neurodegeneration. Parkinsonism Relat Disord 17: 423430 .

30. Navarro-Reig M, Jaumot J, García-Reiriz A, Tauler R (2015) Evaluation of changes induced in rice metabolome by $\mathrm{Cd}$ and $\mathrm{Cu}$ exposure using LC-MS with XCMS and MCR-ALS data analysis strategies. Anal Bioanal Chem 407: 8835-8847.

31. McAllum EJ, Roberts BR, Hickey JL, Dang TN, Grubman A, et al. (2015) Zn II(atsm) is protective in amyotrophic lateral sclerosis model mice via a copper delivery mechanism. Neurobiol Dis 81: 20-24. 